\title{
O STI Progress towards Top-Up Mode Operations at the Advanced Photon Source ${ }^{1}$
}

\author{
L. Emery, M. Borland \\ Argonne National Laboratory, 9700 South Cass Avenue, Argonne, IL 60439
}

\begin{abstract}
The Advanced Photon Source (APS) is a 7-GeV, third-generation synchrotron radiation source. To provide a more stable beam for users, in September 1998 we began commissioning a new operating mode called "top-up." In this mode, the beam current does not decay but is maintained at a high level using frequent injection, while photon shutters are open and photon beams are delivered to users. The exhaustive analysis for top-up safety will be reviewed as well as the hardware and software required for top-up operation. Operational experience so far includes testing aspects of top-up injection, delivering beam to X-ray users for a few hours with fractional current stability of 0.001 , and routinely providing beam to users by refilling the ring to $100 \mathrm{~mA}$ every 12 hours with shutters open. Top-up performance issues encountered are shortlived orbit and emittance transients during the injection event, which appear in user experiments as X-ray beam brightness dips. Planned system modifications to reduce these beam transients are described. The main operational issue left for continuous top-up injection will then be sharing the injector system with other operations.
\end{abstract}

\section{INTRODUCTION}

Top-up injection refers to injecting with photon shutters open to deliver a nearconstant stored beam current. This will improve X-ray beam stability through a constant heat load on X-ray optics and eliminate current-dependent systematics of storage ring (SR) beam diagnostics.

A relative current stability of $10^{-4}$ is our long-term goal. We have acheived this for a few hours of running during machine studies. A lower stability of $10^{-3}$ was achieved routinely, and has been delivered to users for several hours on a trial basis.

An equally important beam quality issue for top-up is the beam disturbance that may be caused by the injection process. Though any closed orbit or emittance disturbance is damped out after several tens of milliseconds through synchrotron radiation damping or the decay of pulsed magnet fields, these disturbances may

1) Work supported by U.S. Department of Energy, Office of Basic Energy Sciences, under Contract No. W-31-109-ENG-38. 


\section{DISCLAIMER}

This report was prepared as an account of work sponsored by an agency of the United States Government. Neither the United States Government nor any agency thereof, nor any of their employees, make any warranty, express or implied, or assumes any legal liability or responsibility for the accuracy, completeness, or usefulness of any information, apparatus, product, or process disclosed, or represents that its use would not infringe privately owned rights. Reference herein to any specific commercial product, process, or service by trade name, trademark, manufacturer, or otherwise does not necessarily constitute or imply its endorsement, recommendation, or favoring by the United States Government or any agency thereof. The views and opinions of authors expressed herein do not necessarily state or reflect those of the United States Government or any agency thereof. 


\section{DISCLAIMER}

Portions of this document may be illegible in electronic image products. Images are produced from the best available original document. 
still affect some X-ray experiments. We will report on steps taken to reduce the impact of these injection transients on $\mathrm{X}$-ray experiments.

\section{RADIATION SAFETY}

Photon shutters are normally closed during injection to block any injected beam particles from escaping the SR enclosure and entering the experiment hall, where they would constitute a radiation hazard. (This might occur, say, due to a short in a dipole magnet with a photon port.) Prevention of such an accident is the main safety issue in top-up and was the subject of extensive studies $[1,2]$.

We found that if the dipole magnets and power supplies are operating normally, no configuration error or magnet fault can produce an accident. Simulations gave a stronger conclusion, that even with a shorted dipole and other errors, one cannot have stored beam while extracting injected beam down a photon beamline. Thus, we ensure the top-up safety with an interlock that inhibits injection with shutters open if there is no stored beam.

The simulations used a series of lattices and conservative (i.e., accidentenhancing) choices of magnet faults. All scenarios involved a shorted dipole of variable degree. Other faults considered simultaneously were hypothetical mis-set quadrupoles, worst-case dipole steering from malfunctioning nearby multipoles, and injected beam energy error. To limit the possible steering from multipoles, we ran the simulations for $6 \mathrm{GeV}$; a hardware interlock on the dipole power supply enforces this minimum energy.

Because of the importance of aperture location and dimensions in limiting the possible trajectories of the injected beam, we require controlled drawings and documents listing the relevant apertures (those used in the tracking simulations). Tolerances for the placement of apertures (determined from tracking) are also documented. Routine checks of the placement of apertures are required. Since apertures in the SR vacuum chambers and photon beamline are not visible from the outside, this is done indirectly using survey measurements of the magnets and photon beamline safety shutter, and using measurement gauges to verify the position of vacuum chambers in magnets.

\section{OTHER OPERATIONAL CONCERNS}

The injector produces a single bunch at $2 \mathrm{~Hz}$, with a nominal charge of $1 \mathrm{nC}$ and design maximum of $20 \mathrm{nC}$. For $10^{-4}$ current stability, the injected charge is about $0.04 \mathrm{nC}$, at the bottom range of the beam transport line diagnostics sensitivity. Beam diagnostics with higher sensitivity are planned. In the meantime, scrapers in the transport line can be used to scrape down to the required low charge.

Permanent magnets in insertion devices (IDs) can be demagnetized by a large radiation dose. Injected beam losses are highest at the ID vacuum chambers (VCs). In top-up operation the IDs are closed, almost touching the VCs. Shielding cannot reduce the dose at the downstream end of the ID since a radiation shower travels 
inside the beam pipe and through the thin $(1 \mathrm{~mm}) \mathrm{Al}$ chamber. Injection losses will have to be closely monitored. A Cerenkov detector has been installed on one ID VC to serve as a beam loss diagnostic. We plan to equip all ID VCs similarly.

SR injection uses a four-magnet closed kicker bump lasting less than one turn, as well as two septa at the end of the transfer line. Poor injection efficiency is typically due to variation of injected beam initial coordinates in the SR that causes particles to hit vertical or horizontal apertures, which are both small because of small gap undulator apertures (the smallest aperture is $\pm 2.5 \mathrm{~mm}$ in $y$ and $-15 \mathrm{~mm}$ in $x$ ). Automatic slow trajectory correction controls the vertical and most of the horizontal motion. However, pulse-to-pulse jitter of the pulsed magnets produces horizontal motion that cannot be corrected. Improved pulsed supply performance, increased injection aperture, and reducing the booster beam emittance should reduce injection losses. With a recent improvement of injection aperture (by swapping out a septum that had a large weld on a beam pipe) the injection efficiency is nearly $100 \%$ with a closed kicker bump. In the meantime, virtually $100 \%$ injection efficiency can be obtained using a mismatched kicker bump that reapportions the betatron oscillation of the injected beam between the injected beam and the stored beam.

\section{BEAM PERTURBATION}

The injection process is required to be transparent during user experiments. Stored beam centroid motion or increased emittance will reduce the X-ray brightness. The mismatched injection bump, which ensures good injection, perturbs the stored beam, imparting a betatron amplitude of a few millimeters. Decoherence (due to tune spread) occurs in about 20 turns, replacing the centroid motion with increased emittance. A six-fold increase in measured beamsize in a single bunch is seen after injection and lasts a few damping times, during which time the synchrotron radiation brightness is greatly reduced. The beam returns to normal in about $30 \mathrm{~ms}$. The blow-up is expected to be greatly reduced when aperture problems are fixed, which will allow a closed kicker bump.

The next problem is the accurate matching of the kicker magnetic fields for the duration of the pulse. Being of the same design, the kickers produce magnetic fields with almost the same rise and decay times. However, a few percent difference will induce a significant betatron oscillation $(\sim 2 \mathrm{~mm})$ in some stored bunches and thus create an emittance blow-up for part of the beam. The same effect can be caused by the nonlinear fields of sextupoles inside the bump, though the sextupole strength distribution can be optimized to be self-compensating. We are presently making adjustments to the kicker power supply hardware.

Another injection-related beam perturbation is a closed-orbit distortion produced by the leakage field of a pulsed H-type dipole magnet at the end of the injection line. This magnet, pulsed with a $10-\mathrm{ms}$ half sine wave, is very close to the stored beam as it almost touches the SR vacuum chamber. Various beamlines will experience different orbit and angle distortion depending on the phase advance from the septum. The beam disturbance lasts for a total of about $25 \mathrm{~ms}$, and the maximum 
orbit distortion is about $200 \mu \mathrm{m}$-about $60 \%$ of the horizontal beam size. The present pulsed magnet replaced the original one that had a solid-core end pack and coil connection positioned at the exit, both of which contributed to a $600-\mu \mathrm{m}$ leakage field orbit distortion.

The leakage-field-induced closed-orbit distortion will be corrected using the realtime orbit feedback system switched to a feedforward mode in a time window around the injection transient. While this system cannot react quickly enough in feedback mode to completely eliminate the distortion, it is expected that in feedforward mode the system's $1.6-\mathrm{kHz}$ update rate should suffice. The new feedforward mode of the orbit feedback system will be tested in October 1999.

Injection-related trigger signals are available to X-ray experimenters so that Xray data acquisition can be blocked during a time window around the injection event. This is most useful for counting experiments. The possible blanking out of $35 \mathrm{~ms}$ of beam time every two minutes does not reduce the average flux significantly, as it provides a $99.97 \%$ duty factor.

Those experimenters taking data by CCD camera imaging require uninterrupted beam for a wide range of time intervals, say 1 second to 30 minutes. Any beam disturbance that reduces X-ray brightness during a long CCD camera scan may produce an unacceptable spurious signal. Since our current injection process produces a beam perturbation, in the short term we must adopt a top-up injection time interval longer than the longest CCD camera experiment, say one to several hours following a strict agreed-upon schedule. This mode does not provide all the advantages of top-up, but can significantly increase X-ray availability.

\section{COMMISSIONING}

Commissioning of top-up operation mode involves injecting beam in the SR with the photon shutters open for the first time. The commissioning plan entails measuring radiation dose rates outside shielding walls in areas possibly occupied by personnel for various beam loss scenarios and checking that the measured doses are within acceptable values. The beam loss scenarios considered here are those allowed by the personnel protection system. The first scenario is an injected beam loss inside the SR enclosure; the second, an injected beam missteered at an ID vacuum chamber.

The maximum radiation hazard is expected to be produced when the injected beam is missteered into an ID vacuum chamber aperture. Bremstrahlung photons travel through the open shutters and scatter in the first optics enclosure (FOE), producing a dose outside the FOE. In the year leading up to commissioning, measurements were done simulating misteering by repeatedly dumping a stored beam on different ID VCs with the corresponding beamline shutters open. The worst results were scaled to the injection safety envelope of $20 \mathrm{nC} / \mathrm{s}$, giving a maximum credible incident value of $6.3 \mathrm{rem} / \mathrm{h}$.

The above bremsstrahlung radiation measurement was repeated with an actual $1-\mathrm{nC} / \mathrm{s}$ injected beam continuously dumped on two separate ID vacuum chambers with corresponding beamline shutters open. The largest dose measured around the 
FOE adjusted to the safety envelope conditions was $0.25 \mathrm{rem} / \mathrm{h}$. The variability of the equipment housed in the FOEs may explain the discrepancy between these two experiments.

Assuming a $100-\mathrm{mA}$ beam with 10 hour lifetime, a current stability of $10^{-3}$ requires injection of $0.37 \mathrm{nC}$ every 36 seconds. If every pulse was lost on an ID VC, the maximum dose rate outside the SR tunnel would be about $3 \mathrm{mrem} / \mathrm{h}$.

The tracking studies of top-up safety cannot be verified easily as it would involve defeating the personnel protection system and creating difficult-to-generate magnet faults. However, we verified one aspect of the simulations. The tracking studies give a conservative limit for the negative fractional strength error (FSE) for a dipole magnet that would preclude stored beam. The tracking limit, FSE $=-0.09$, would be supported if the measured limit was smaller in absolute value. Dipole trim coils were used to simulate a negative FSE in a particular dipole. Beam was lost at $\mathrm{FSE}=-0.050(-0.036)$ with (without) orbit correction running.

\section{TOP-UP OPERATION}

There are two top-up operation modes, a high current-stability mode (frequent injection) and a refill mode (injection every few hours). The relative current stability, $\epsilon$, is defined by $\left|I(t)-I_{0}\right|<\epsilon I_{0}$, where $I(t)$ is the instantaneous current and $I_{0}$ is the target current.

In high current-stability mode we could either inject on an as-needed basis or at a constant time interval. For the former, injection occurs when the beam decays below $I_{0}$. Because of typical $10 \%$ injector performance variation, injection occurs at irregular intervals. Injecting with $0.04-\mathrm{nC}$ charge, we achieved $10^{-4}$ stability for $I_{0}=100 \mathrm{~mA}$, with an injection interval of about $7 \mathrm{~s}$. A $3 \times 10^{-4}$ current stability was achieved by injecting $1 \mathrm{nC}$ every few minutes.

Since the unpredictable time of the injection event in this mode is undesirable to users who are sensitive to the injection process, we developed a fixed-injectioninterval mode. One specifies an injection interval $T$ and a target current $I_{0}$, and sets up the injector to deliver enough charge $\left(\Delta I=I_{0} T / \tau\right)$ to replace the beam lost through natural scattering processes ( $\tau$ is beam lifetime). At the end of the interval, injection occurs only if $I<I_{0}$, giving stability of $2 \Delta I$.

In the refill top-up mode the beam is allowed to decay undisturbed for several hours, then beam is injected to reach the target current. For example, in one hour the $100-\mathrm{mA}$ beam decays by 3 to $5 \mathrm{~mA}$, depending on the bunch pattern. Refilling every hour with the APS injector running at $2 \mathrm{~Hz}$ and $0.8 \mathrm{nC} /$ pulse would take 8 to 12 seconds, giving a high duty factor $(99.7 \%)$ and a low user impact. The present standard APS operation involves filling to $100 \mathrm{~mA}$ every 12 hours. The refilling time is about 90 seconds.

In top-up injection with high current stability, only one pulse in one SR bucket is injected. In general, a different bucket is selected at every pulse to maintain the bunch pattern as well as the total current. A constant bunch pattern is desirable for beam position monitor triggering and offset stability, and for X-ray user timing experiments. 
The target buckets are selected between top-up injection events, during which time the bunch pattern intensity is measured, averaged, and compared with a reference bunch pattern intensity. The bunches that need charge the most will receive the charge of the next injection sequence. The stability of the bunch charge is of the order of $\epsilon$ times the number of bunches. For example, for a bunch pattern of about 25 bunches, the bunch current variation could be as high as $2 \%$.

We measured the jitter in the timing of the injection pulses in response to user concerns of possible mistimed injection pulses. We found no missed or skipped injection pulses in several hours of testing and measured the jitter in time to be $60 \mathrm{~ms}$ in a one-hour measurement [3]. This jitter is consistent with the $60-\mathrm{Hz}$ line frequency drift.

We plan to implement pulse histories for the SR pulsed magnets, particularly in the kickers, in order to help diagnose possible beam dumps during top-up. Though the kickers have been very reliable, and we have no evidence of kicker-caused beam dumps in the past, it would be useful to be able to eliminate this possibility when diagnosing causes of beam dumps.

During the 12 hours between refills of our present operating mode, the injector system is available for other applications. Specifically, the linac beam and the lowenergy transport line are used to provide beam for the low-energy undulator test line (LEUTL) in support of a free-electron laser (FEL) demonstration program. Presently switching between the two operating configurations, each optimized for its particular application, requires some 10 minutes of setup time. Our ultimate goal is to make true top-up mode (high current stability) compatible with operating the LEUTL. This involves switching quickly between the two injector configurations such that linac beam pulses can alternate between FEL studies and SR injection, with the SR injection expected to occur about once a minute and the LEUTL beam to be virtually uninterrupted. An area for development is making dipole and quadrupole magnet power supplies and rf system parameters switch between two setpoints within 0.5 seconds or less.

\section{ACKNOWLEDGEMENTS}

The authors wish to acknowledge the work of all the techical groups at APS for making top-up injection possible.

\section{REFERENCES}

1. L. Emery, M. Borland, "Analytical Studies of Top-Up Safety for the Advanced Photon Source," Proceedings of 1999 Particle Accelerator Conference, pp. 2939-2941.

2. M. Borland, L. Emery, "Tracking Studies of Top-Up Safety for the Advanced Photon Source," Proceedings of 1999 Particle Accelerator Conference, pp. 2319-2321.

3. F. Lenkszus, R. Laird, private communication. 


\section{APS ACCELERATOR SYSTEMS DIVISION}

Report: $\quad$ Final Paper-SRI'99

Title: $\quad$ "Progress Towards Top-Up Mode Operations at the Advanced Photon Source"

\section{Signature Approval Date}

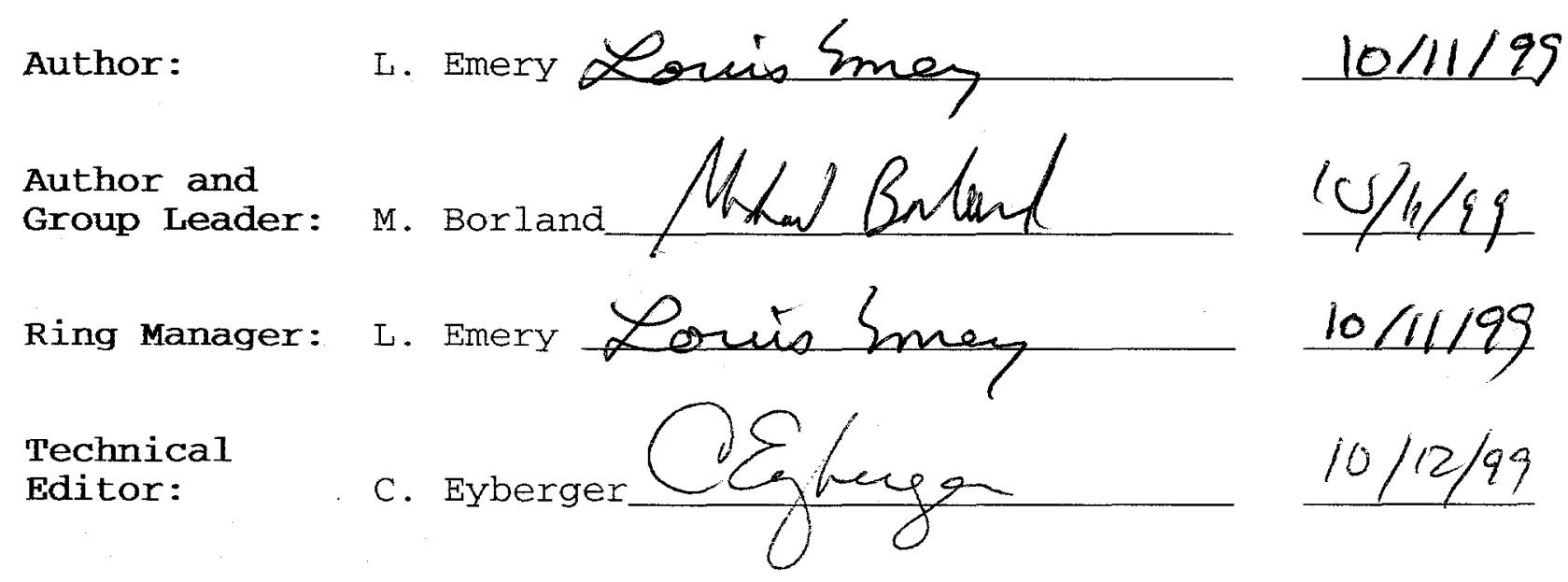

When you have completed your review of the subject paper, please sign in the appropriate space and return to Mary Kay Jakovich, 401-B2147C.

Thank you. 